\title{
A Comparison of Behavioral Activation/Inhibition Systems and Mindfulness in Addicts and Non-addicted Males
}

\author{
Gholam Hossein Javanmard ${ }^{1}$, Masumeh Mirhasanpour Vahedi ${ }^{2,}$, \\ ${ }^{1}$ Department of Psychology, Payame Noor University (PNU), Tehran, Iran \\ ${ }^{2}$ Department of Psychology, Science \& Research Branch of Islamic Azad University of Tabriz, Tabriz, Iran
}

Email address:

mirhasanpourm@gmail.com (M. M. Vahedi)

*Corresponding author

\section{To cite this article:}

Gholam Hossein Javanmard, Masumeh Mirhasanpour Vahedi. A Comparison of Behavioral Activation/Inhibition Systems and Mindfulness in Addicts and Non-addicted Males. Psychology and Behavioral Sciences. Vol. 6, No. 3, 2017, pp. 43-48. doi: 10.11648/j.pbs.20170603.12

Received: April 20, 2017; Accepted: May 8, 2017; Published: June 23, 2017

\begin{abstract}
The main objective of this causal-comparative research was to compare behavioral activation and inhibition systems and mindfulness in addicts and non-addicted men of Maragheh, Iran. In order to do this, 35 addicted males and 35 non-addicted men were chosen using purposeful sampling method and via matching the two groups. The participants answered the 120-item test of activation/inhibition systems (Gray-Wilson Personality Questionnaire or GWPQ) and the 39-item test of mindfulness (Kentucky Inventory of Mindfulness Skills or KIMS). Data were analyzed using multivariate analysis of variance (MANOVA) and SPSS software. The findings showed that the compared groups in activation/inhibition systems and mindfulness had meaningful differences. The average scores of mindfulness in healthy people were higher than those of the addicted people. While the average scores of behavioral inhibition system in healthy people were higher than addicts, the average scores of behavioral activation system in addicts were higher than non-addicted people. In general, since this study showed that activity level of activation system in addicted people is more than non-addicts, activity level of inhibition system in non-addicts is more than addicted people, and that mindfulness skill in addicts is lower than non-addicts there is the necessity to design therapeutic and rehabilitative interventions.
\end{abstract}

Keywords: Activation/Inhibition Systems, Mindfulness, Addiction

\section{Introduction and Literature Review}

Drug addiction is one of the major problems that has caused a lot of serious problems and disasters for the human societies. Drug addiction is a chronic and recurrent disorder imposing heavy costs on both the individual and society. It also results in intense motivational disorders and personality destruction. The spread of drug use among young people is higher than any other age group. Drug use and abuse is one of the riskiest behaviors during childhood, adolescence and adulthood [29].

Decades of research have proved that continuous drug abuse may damage normal functioning of cerebral cortex and its rewarding system causing deviations in brain's reward system, surfaces of information processing and neurotransmitter areas in the brain. Different views sought to explain the individual differences through biological variables, among them is Pavlov who introduced two fundamental processes that govern all activities: stimulation and inhibition. Besides, Gray's Reinforcement Sensitivity Theory (RST) is a biological perspective on personality that sought to explain, before Eysenck, the uncontrollable desire of individuals for drug abuse [13]. According to Gary, the Behavioral Inhibition System (BIS), Behavioral Activation System (BAS) and Fight-Flight-Freeze System (FFFS) are the bases of individual differences [28].

While BIS responds to conditioned stimuli that are associated with punishment or removal of reward and leads to arousal and behavioral avoidance, BAS responds to stimuli associated with reward or removal of punishment and leads to arousal and behavioral approach. Many studies have showed that BAS is a risk factor for drug tendency. Smoking plays an important role. BIS, BAS and FFFS are the bases of individual differences and the activity of each of these behavioral brain systems provokes different emotional 
reactions in individuals [25], [10].

A comprehensive study in Iran, for the first time, compared behavioral systems and mental health of addicts and non-addicts. The results of this study showed that the activity of BAS in the addicts group was more than normal group, and average BIS scores of normal people were higher than addicts [3]. Other research findings suggest that both components of BAS in addicted males and tendency scale in addicted females are more active than non-addict peers [28]. The results consistent with other findings suggest that increasing levels of impulsivity and behavioral activation system, and reduction in self-efficacy, behavioral inhibition and social skills increase drug abuse among students [27]. Recent studies in the field are consistent with the previous findings, i.e., while BAS is more active in males, BIS is more active in females [24].

Various studies showed that there is a positive relationship between drug abuse and high activity of BAS, and a negative relationship between drug abuse and BIS [13], [17], [19]. There are similar results in Iran showing that drug abusers and smokers are more BAS-sensitive compared to others which makes them susceptible to tendency behavior [1]. It should also be mentioned that Hasking's research (2006) is one of the few studies that couldn't find any relationship between drug abuse and sensitivity of BAS. Moreover, Loxton and Dawe (2006) and Loxton et al (2008) did not find any relationship between inhibition systems and drug abuse.

The complexity of the factors affecting the treatment and control of drug addiction has caused different groups of researchers to examine it from specific perspectives. Recently, the psychological interventions approach of "mindfulness" has helped us to understand that although negative emotions and events occur during life, but they are not fixed parts of the character and life of an individual. Hence, this makes the individual respond and react with contemplation rather than responding involuntarily to these events, therefore, mindfulness is a new style of communicating more effectively with life which relieves pains and sufferings and makes life enjoyable and meaningful [2]. Mindfulness has also been used in the field of drug addiction with positive treatment results and effects. If mindfulness is to be combined with preventive methods, it would have relatively successful effects on judgment and aversion to drugs in injection drug users [30]. Temptation is the strongest predictor of relapse among other predictors. Mindfulness can reduce the negative effects of temptation considerably. Researchs show that mindfulness is a useful incremental strategy to reduce the likelihood of recurrence.

Using mindfulness approach yielded good results in therapeutic interventions of drug addiction and relapse prevention. Training relapse prevention model is effective on addiction and motivation [20]. Moreover, mindfulness-based cognitive therapy (MBCT) interventions are more effective than Marlatt's cognitive-behavioral model on the mental health of crack-addicted clients [26]. Teaching mindfulness skills and providing behavioral counseling on AIDS harm reduction and drug use are practically functional for clinical interventions to prevent drug abuse relapse [14]. Acceptance and Commitment Therapy (ACT) and mindfulness, which share common treatment components, are effective treatment scales in reducing psychological trauma caused by the use of amphetamines [18].

Flavel (1979) believes that metacognitive knowledge or cognitive process contributes to evaluation, review and cognitive control and adjusts cognitive function. Metacognition may be particularly associated with cognitive analysis of drug-dependent individuals. From metacognitive perspective, drug abuse brings significant rapid changes in cognitive events such as feelings, thoughts or memories. Narcotic drugs and psychotropic substances may affect cognitive events directly (e.g., for relaxation, avoidance and escape from the painful facts and creating awareness) or indirectly (for example, feel an attachment and shock) by changing beliefs and attitudes. These cognitive changes may be the result of positive and negative reinforcements created by drug use. The sensitivity of BAS (actively searching for reward) and active avoidance (behaving in a certain way to avoid punishment) are some proofs for this issue (Gary Wilson and Barrett 1990). Combination of mindfulness and traditional cognitive-behavioral relapse prevention techniques led to the emergence of third wave of behavior therapy [16], [20], [14]. Mindfulness can reduce symptoms, enhance treatment effectiveness and prevent slipping because it includes concepts such as: acceptance, raising awareness, desensitization, mindfulness, observing without judgment, confrontation and release [33]. Mindfulness can help one in utilizing the biological abilities to benefit himself and his relations by presence in the moment and perception of his own behavioral activation and inhibition. Many questions can be answered by identifying and comparing the characteristics of non-addicts, addicts, and ex-addicts. Few researches have been conducted in this field and there is also a lack of study on activity level of behavioral activation and inhibition systems. This study provides an understanding of addicts and non-addicts by comparing these scales in them. With regards to the aforementioned statements and conflicting findings of studies on BAS and BIS activity level in addicts, this study tries to retest the researches and review the activity level of BAS and BIS in drug addicted and normal individuals. In addition, most researches have studied activation/inhibition systems and mindfulness separately. But this study tries to investigate behavioral activation/inhibition systems of the brain and mindfulness simultaneously in drug addicted and non-addicted individuals.

\section{Method}

The study sample consists of two groups: 1) drug-addicted individuals admitted to drug rehabilitation clinics in the city of Maragheh. This sample includes addicts who were admitted to the rehab centers in 2015 and detoxification was not applied on them. These people had been admitted for less than 4 months and were still addicted to drugs. 2) nonaddicted population (normal people) who were matched with 
the first group.

The sample for this survey was selected from the two communities by purposive sampling. Since the minimum sample size required for causal-comparative studies is 30 subjects from each group [9], the researchers selected 35 male subjects from each group. The inclusion criterion was the ability to read and write; and exclusion criteria were: suffering from mental retardation, chronic physical, mental and neurological diseases and being under drug treatment (except for addiction rehabilitation drugs). Also, both groups were matched for age and educational level.

Tools: the following two questionnaires were used to measure the variables of the study:

1) Gary-Wilson Personality Questionnaire (GWPQ). This questionnaire was designed by Wilson, Barrett and Gary in 1989 and is a self-assessment personality questionnaire that evaluates the activity of behavioral systems and their components. It includes 120 items and each of the questions has three options: "Yes", "No" and "I do not know". "I do not know" option is chosen when a participant can't answer a question with "yes" or "no" at all. In answering every item with a +, "yes" has 2 scores, "I do not know" 1 score and "no" no score. In answering every item with a -, "yes" has no score, "do not know" 1 score and "no" 2 scores. Regarding the validity of the questionnaire, Wilson, Barrett and Gary (1989) reported the Cronbach's alpha coefficients for the components of approach, active avoidance, passive avoidance, extinction and fight-flight-freeze as following: $0.71,0.61,0.58,0.61,0.65,0.65$ for male subjects and 0.68 , $0.35,0.59,0.63,0.71,0.71$ for female subjects. This indicates the good internal consistency of the test. They also used the correlation between GWPQ scales and Eysenck Personality Questionnaire to show the convergent validity of the test. This questionnaire was translated into Persian by Azad Fallah et al (2000) and conducted on a 211-member group of Iranian students. Moreover, Ashrafi (2006) reported the Cronbach's alpha coefficients for the components of approach, active avoidance, passive avoidance, extinction and fight-flightfreeze as following: $0.60,0.54,0.61,0.66,0.65$, and 0.69 . Consistency coefficients through the split-half method were: $0.53,0.57,0.52,0.62,0.64$, and 0.64 respectively (Fathi Ashtiani, 2013).

2) Kentucky Inventory of Mindfulness Skills (KIMS) is a 39-item test designed by Bear, Smith and Allen (2004) for measuring 4 mindfulness components including: observing, describing, act with awareness, and accept without judgment (Golpour, 2011). Test validity was calculated 0.83 by Cronbach's alpha (Golpour, 2011).

\section{Results}

Mean and standard deviation for age of the control group was $31.57+/-6.8$ and for addicted group was $29.83+/-7.6$, and the total was $30.7+/-7.21$. The results of the independent t-test $\mathrm{p}=316, \mathrm{t}(68)=1.01$ show that there is no significant difference between the mean ages of the groups. This means that the groups are matched in terms of age. Also, according to the results of Chi-square test $\mathrm{P}=0.619, \mathrm{X}^{2}=0.25$ both groups were matched in terms of education $(\mathrm{P}>0.05)$.

Table 1. The mean and standard deviation of mindfulness and behavioral activation / inhibition systems (BAS / BIS) in two groups.

\begin{tabular}{lllll}
\hline \multirow{2}{*}{ Variable } & \multicolumn{2}{l}{ Non-addicted Group } & \multicolumn{2}{l}{ Addicted Group } \\
\cline { 2 - 5 } & SD & Mean & SD & Mean \\
\hline Mindfulness & 7.08 & 120.88 & 6.24 & 116.46 \\
BAS & & 3.1979 .54 & 5.38 & 22.37 \\
93.746 .57 & BIS & 35.28 & 6.92 & \\
\hline
\end{tabular}

While non-addicted group shows a higher mean score in behavioral inhibition system, addicted group shows a higher mean score in behavioral activation system. Also, nonaddicted group shows a mean score higher than addicted group in terms of mindfulness.

Table 2. Kolmogorov-Smirnov test results for evaluating the normality of distribution in the two groups.

\begin{tabular}{lllll}
\hline Variable & Group & Statistic & $\begin{array}{l}\text { Degree of } \\
\text { freedom }\end{array}$ & $\begin{array}{l}\text { Significance } \\
\text { level }\end{array}$ \\
\hline Mindfulness & Non-addicted & 0.069 & 35 & 0.200 \\
& Addicted & 0.135 & 35 & 0.105 \\
BAS & Non-addicted & 0.115 & 35 & 0.200 \\
& Addicted & 0.124 & 35 & 0.192 \\
BIS & Non-addicted & 0.146 & 35 & $* 0.051$ \\
& Addicted & 0.074 & 35 & 0.200 \\
\hline
\end{tabular}

Table (2): the significance levels show normal score distribution of mindfulness, BAS and BIS in groups (Significance level $>0.05$ ). To test the homogeneity of covariance matrices, Box's test results were reviewed: significance level of Box's test was 0.172. The significance value $>0.05$ shows that assumption of covariance matrices homogeneity exists.

The homogeneity of variances is one of the assumptions in multivariate analysis of variance which exists according to the reported results of Levene's test. Considering the significance value $>0.05$, the assumption of homogeneity of variance of mindfulness scores and BAS/BIS exists.

The results of implementing multivariate analysis of variance (MANOVA) to compare the two groups in the average linear combination of mindfulness and activation / inhibition systems are presented in Tables 3 and 4:

Table 3. MANOVA results for the effects of group membership.

\begin{tabular}{lllllll}
\hline Test & Value & F & $\begin{array}{l}\text { DF } \\
\text { assumption }\end{array}$ & $\begin{array}{l}\text { DF } \\
\text { error }\end{array}$ & P & $\begin{array}{l}\text { Eta } \\
\text { squared }\end{array}$ \\
\hline Pillai Effect & 0.19 & 5.14 & 3 & 66 & 0.003 & 0.19 \\
Wilks' Lambda & 0.81 & 5.14 & 3 & 66 & 0.003 & 0.19 \\
Red Hotelling & 0.23 & 5.14 & 3 & 66 & 0.003 & 0.19 \\
Greatest root & 0.23 & 5.14 & 3 & 66 & 0.003 & 0.19 \\
\hline
\end{tabular}

MANOVA test results in table 3 show that there is a significant difference between the mean scores of both groups in linear combination of mindfulness and BAS/BIS $(\mathrm{P}<0 / 01)$. 
Table 4. Multivariate ANOVA for comparing the group means in mindfulness and BAS/BIS.

\begin{tabular}{llllll}
\hline Variables & Sum of squares & Degrees of freedom & Mean square & F & Significance level \\
\hline Mindfulness & 343.21 & 1 & 343.21 & 7.71 & 0.007 \\
BAS & 140.01 & 1 & 140.01 & 6.46 & 0.013 \\
BIS & 91.43 & 1 & 91.43 & 1.33 & 0.253 \\
\hline
\end{tabular}

The data provided in Table 4 and univariate comparisons show that two groups are significantly different in terms of mindfulness. For this variable, the value of $F$ is 7.71 , etasquared is 0.10 and significance level is less than 0.01 . Given that the size of significance level is less than 0.05 in table 2 and by considering the mean of mindfulness in the control group (120.88) and addicts (116.46), in table 1, it is inferred that addicts have weak mindfulness skills compared to nonaddicts. Also, univariate comparison between the two groups shows that they are significantly different in terms of BAS. For this variable, $\mathrm{F}$ is 6.46 , eta-squared is 0.09 and significance level is less than 0.05 . Given that the size of significance level is less than 0.05 in table 4 and by considering the mean of BAS in the control group (19.54) and in addicts (22.37), in table 3, the mean of BAS in addicts is significantly greater than that of non-addicts.

\section{Discussion}

This study made an attempt to find existing relations and interactions between biological factors of behavioral systems and self-monitoring factor in individuals' tendency towards drug use. Thus, according to theoretical models and empirical research, we can conclude that personality traits play a role in all processes of tendency towards drugs, addiction and rehabilitation. The results of most studies indicate that the activity level of BAS in the addicts group is more than nonaddicts, and the mean scores of BIS in normal group are higher than those of the addicts. Also while BAS is more active in men compared to women, BIS is more active in women. This study showed that inhibition-activation systems and mindfulness in addicts is different from non-addicts namely, BAS is more active in addicts than non-addicts, and mindfulness level in addicts is lower than non-addicts. Numerous studies have shown the role of early temptation to drug abuse [6]. High reactivity leads to negative emotion regulation strategies and thus increases drug use.

Other research findings show that both components of BAS in addicted men and approach component of BAS in addicted women are more active compared to non-addicted peers [28]. Results consistent with other findings suggest that increasing levels of impulsivity, behavioral activation system, and reduction in self-efficacy increases behavioral inhibition and social skills in drug abuse among students [27]. Recent studies in the field are consistent with the previous findings and show that BAS is more active in males than females and BIS is more active in females than males [24].

Franken and Murris (2006) conducted a study about the relationship between personality traits and drug abuse on 276 university students. Results showed that alcohol and drug use by students is positively associated with BAS personality traits, and to some extent, negatively correlated with BIS personality traits. The highest real correlation was found between BAS, the number of unauthorized materials that the person had consumed, amount of alcohol consumption and repetitive and periodic drinking. Comparing the correlation coefficients showed that looking for entertainment (BAS) has a significant relationship with drug abuse. In the field of addiction, most attention is focused on behavioral activation system and most of the findings on the activity of the system in human stems from the studies related to the dopamine neurotransmitters. The release of dopamine in the dopaminergic pathways related to behavioral activation system is associated with the movement programs of this system [4]. In terms of behavior, evidences indicate that the release of dopamine in the normal processes results in establishing and maintaining a chain of specific tool responses which end in food, water and so on [4]. On the other hand, it is also emphasized that use of drugs such as heroin, cocaine, amphetamines, alcohol and nicotine causes the release of dopamine in the above-mentioned neural pathways (Mitchell, 1989; as cited in Azad Fallah, 2000). The low tolerance for discomfort, lack of sleep, stress, anxiety disorders, and low level of mindfulness which are all symptoms of temptation are involved in substance abuse. Mindfulness training can increase the cognitive control on temptation and reduce the stress associated with drug abuse. Also, emphasizing on acceptance instead of suppressing thoughts and breaking the stress-relapse chain in substance abuse may increase the recovery rate. Ly and Gomez (2014) investigated the relationship between brain behavioral systems and anxiety, observation and social interaction showed that both forms of anxiety have a positive relationship with sensitivity to punishment and behavioral inhibition system, but they are negatively correlated with behavioral activation system. Results of a study by Vervoort, Wolters, Hogendoom, De Han, Boer and Prins (2010) confirmed the assumption that clinical anxiety is associated with high activity of BAS. In this study, scores of BIS in anxious group were higher than those of the non-anxious.

\section{Conclusion}

Our findings suggest that drug abuse can be regarded as a way of coping with stress and the pressures of life. Psychological interventions are an inevitable part of treating drug addiction. These methods provide addicts with motivation, increasing social support and coping skills in dealing with the problems associated with drug use. Mindfulness training improves the quality of life in addicts 
both physically and psychologically. Also, mindfulness as one of the main methods of acceptance-based strategies causes the patient to accept his own distressful thoughts and feelings without any judgment or evaluation, instead of challenging and avoiding distressful thoughts and feelings of quitting drug use or use of drugs to relief pains. This is fulfilled via using a different communication style and a new way of processing information. In this way, he may cope with them through desensitization. Some of these changes may be due to mechanisms of action proposed in mindfulness techniques such as confrontation, acceptance, relaxation, desensitization, changing communicating with thoughts and emotion regulation [7]. Awareness of emotions provides ability to identify and control those emotions. Studies have shown that learning such patterns as mindfulness and dealing with emotional and social situations play a role in the prevention and treatment of substance abuse. Children with special nature and tendency like excessive excitation or severe inhibition may feel more anger and frustration when they are in a position to solve a problem. So, they are more susceptible to deal with these emotions by drug use. On the contrary, teens' tendency to substance abuse declines when they learn how to assess and solve their problems more effectively [32].

Training mindfulness skills to patients with substance abuse helps them to be present in the moment and gain knowledge of themselves and the current situation so they can resist the reactivity of activation system and utilize their biological abilities to benefit themselves and their interactions.

\section{References}

[1] Abdi, Reza; Bakhshipour Roodsari, Abbas; and Mahmoud Alilo, Mohammad. (2011). sensitivity of the tendency and behavioral inhibition system on drug addicts, smokers and non-smokers, Iranian Journal of Psychiatry and Clinical Psychology, 17 (3), 247-241.

[2] Ahmadvand, Zahra; Heidari Nasab, Leila, Shaieri, Mohammadreza (2013). A study of the validity and reliability of five-dimensional mindfulness questionnaire in non-clinical Iranian samples. Journal of Behavioral Sciences, 7 (3), 229-237.

[3] Alimoradi, Abdul Latif; Hooshyar, Samaneh; Modarres Ghoravi, Morteza. (2011). Comparing the brain-Behavioral Systems and mental health in drug addicts and non addicts. Journal of Mental Health, 13 (4), 13-304

[4] Azad fallah, Parviz, Dadsetan, Parirokh (2000). Brain / Behavior systems: biological structures of Personality, Journal of Psychology, Volume 4, Issue 1, pp. 82-63. Azad fallah, c. (2000). Ecological- psychological basis of addiction. Journal of Psychology, no.3, 246-234.

[5] Babapour, Khairaddin; Dadashzadeh, Rahim, Toosi, Fahimeh. (1390), "comparing smokers' and nonsmokers' behavioral system. Journal of Modern psychological research year 6, no. 23, Summer 2011.

[6] Bjork, J. M., Hommer, D. W., Grant, S. J., \& Danube, C. (2004). Impulsivity in Abstinent Alcohl-Dependent Patients:
Relation to Control Subjects and Type 1-/ Type 2- Like Traits. Alcohol, 34(1), 133- 150.

[7] Breslin, F., Zak, M., MC Main, S. (2002). An information processing analysis of mindfulness: Im placations for relapse prevention in the treatment of substance abuse. Clinical Psychology: Science and Practice, 9(3): 275-99.

[8] Dabaghi, P.; Asgharnejad Farid, Ali; Atef Vahid, Asghar; MohammadKazem and Bolhari, Jafar. (2008), "The effectiveness of group cognitive therapy based on mindfulness (mindfulness) and spiritual schema activation in the prevention of relapse in opioid abuse." Journal of thought and behavior, No. 4, pp. 375-366.

[9] Delavar, Ali (2010). Research methods in psychology and Educational science, Tehran, published and edited.

[10] De Pascalis, V.; Varriale, V.; \& D Antono, 1. (2010). "Eventrelated components of the punishment and reward sesitivity" Clinical Neurophysiolojy. 121 (1), 60-76.

[11] Desjardins, J., Zeleneski, J. M., \&Cpplan, R. J. (2008). An Investigation of Maternal Personality, Parenting and Subjective Well-Being, Personality and Individuol Differences, Vol.44:587-597.

[12] Flavell J. Metacognition and cognitive monitoring: A new area of Cognitive developmental inquiry. Am Psychol. 1979; (34):906-11.

[13] Franken I H, Muris P.(2006) BIS/BAS Personality characteristics And College Students Substance us. Personality IndivDiffer, 40:1497-503.

[14] Hamedi, Ali; Shahidi, Shahryar; Khademi, Ali. (2013). the effectiveness of mindfulness and advice on the prevention of relapse Journal of Substance Abuse Addiction Studies, 7 (28).

[15] Hasking PA, (2006) Reinforcement Sensitivity, Coping DisorderedEating and Drinking Behaviors in Adolescents. Pers IndiveDiffer,40:677-88.

[16] Hayes, S., Luma, J. B., \& Bond, F. (2006). Acceptance andcommitment therapy: Model, Processes andoutcomes. Behav Res Ther, 44, 1- 25.

[17] Hundt, N. E., Kimbrel, N. A., Mitchell, J. T., \& Nelson-Gray, R. O. (2008). High BAS, but not low BIS, Predicts extemalizing symptoms in adults. Personality and Individual Differences, 44(3),563-573.

[18] Kayani, AR; Ghasemi, Nizamuddin; Pourabbas, A. (2012). Comparing the effectiveness of group therapy, acceptance and commitment therapy based on cognitive emotion regulation in consumer awareness on glass addicted. Journal of Substance Abuse Addiction Studies, 6 (24).

[19] Kimbrel, N. A., Nelson- Gray, R. O., \& Mitchell, J. T. (2007). Reinforcement sensitivity and maternal style us predictors of psychopathology. Personality and Individual Differences, 42(6), 1139-1149.

[20] Klori, Ali; Borjali, Ahmed; Falsafinejad, MR; Sohrabi, F. (2011). Effectiveness of model of relapse and temptation prevention in drug addicted. Journal of Clinical Psychology, 3 (4).

[21] Loxton, N. J., \&Dawe, s. (2007). Howdodys functional eating and hazardous drinking women perform on behavioral measures of reward and punishment sensitivity? Personality and Individual Differencees, 42(6), 1163-1127. 
[22] Loxton N. J., DAW S. (2008) Reward and Punishment Sensitivity Dysfunctional eating and hazardous drinking women: Associations with family risk Appetite, 47:361-71.

[23] Ly, C., \& Gomez, R. (2014). Unique associations of reinforcement sensitivity theory dimensions with social interaction anxiety and social observation anxiety. Personality and Individual Differences, 60, 20-24.

[24] Maleki Gamchy, A; Khademi, A. (2014). Comparing the Behavioral Systems BAS $\backslash$ BIS in drug-dependent and normal individuals. Police Medicine Journal, 3 (2), 67-67.

[25] Oconnor, R. M., Stewart, S. H., \& Watt, M. C. (2009). Distinguishing BAS risk for university students drinking, smoking, and gambling behaviors. Personality and Individual Differences, 46(4), 514-519.

[26] Orki, Muhammad, Bayat Shohreh; Khoda Dost, S. (2012), "Assessing the effectiveness of cognitive-behavioral intervention model based on Marlatt with mindfulness-based cognitive therapy effectiveness and treatment of drug addicted mental health seekers" Journal of Studies / no.2 - summer 2012.

[27] Pourkord Mahdi, Abolghasemi, Abas; Narimani, Mohammad, Rezaei Jamaloie, H. (2013). The direct or indirect effects of self-efficacy, impulsivity, activation of social behavior inhibition in drug adicted students. Journal of Substance Abuse Addiction Studies, 7 (26).
[28] Pourmohseni Klori, Fereshteh; Haghshenas, Azadeh; Asadi, Saba (2011). Examining brain-behavioral systems and gender differences in addicted and non-addicted. New Cognitive Science, 13 (2), 9

[29] Rutter, M. (2002). Substance Use and Abuse: Causal Pathways Considerations, In M. Rutter \& Taylor (Ads) Child and And Adolescent Psychiatry Models Approaches, Oxford: Blackwell Scientific, 455- 462.

[30] Treloar, C., Laybutt, B., \& Carruthers, S. (2010). Using mindfulness Develop healt education strategies for blood bome virus Prevention in injecting drug use. Drugs: Education, prevention, And Policy, 17(4): 431-42.

[31] Vervoort L., Wolters, L. H., Hogendoom, S. M. DC Huan, E., Boer, F. \&Prins, P. J. M. (2010).Sensitivity of Gray WWW.Science direct.Com/Science article/ Pii/ So 1918869100000 non-anxious children and adolescent. Personality and Individual Differences, 48(5), 629-633.

[32] Windle, M., \& Windle, R. C. (2006). Adolescent temperament and lifetime psychiatric and substanceabuse disorders assessed in yang adulthood. Personality and Individual Differences, 41(1), 15-25.

[33] Witkiewitz, K., Marlatt, GA., \& Walker D. (2005). Mindfulness-based relapse Prevention for alcohol and substance use disorders. Journal of Cognitive Psychotherapy, 19(3):211-28. 